\title{
Efecto antihipertensivo del eprosartan en pacientes diabéticos
}

\author{
N. R. ROBLES, J. VELASCO, J. L. JIMÉNEZ PÉREZ, F. J. ALONSO, J. BLANCO, \\ J. MASOT, C. MATILLA, J. NIETO, F. ROMERO, M. J. GAMERO \\ Unidad de Hipertensión Arterial. Hospital Infanta Cristina. Centros de Salud. Badajoz
}

\author{
ANTI-HIPERTENSIVE EFFECT OF EPROSARTAN IN DIABETIC \\ PATIENTS
}

\section{RESUMEN}

Objetivo: La presencia de diabetes mellitus parece conferir una dificultad especial en el control de la HTA incidiendo particularmente sobre el componente sistólico de la PA. Estudios previos sugieren que un nuevo antagonista de los receptores de angiotensina, eprosartan, podría tener una mayor eficacia en la reducción de la PAS. Se ha evaluado de forma comparativa la reducción de la PA obtenida con eprosartan en un grupo de pacientes diabéticos y sin diabetes mellitus.

Material y métodos: Se reclutaron para el estudio 81 pacientes de los cuales 65 habian terminado el periodo de seguimiento. De ellos, 34 pacientes eran diabéticos (edad 66,7 $10,7,15$ hombres y 19 mujeres) y 31 no padecian diabetes (edad $61,8 \pm 12,8,13$ varones y 18 mujeres). Todos los pacientes fueron tratados con eprosartan $(600 \mathrm{mg})$ en dosis única matutina. Se realizaron visitas de revisión al mes, a los 3 meses y a los 6 meses de la primera consulta.

Resultados: La PAS se redujo significativamente tanto en el grupo de diabéticos ( inicial 170,9 $\pm 12,0$, final 139,1 $\pm 13,0 \mathrm{mmHg}, \mathrm{p}<0,001$ ) como en el grupo sin diabetes (inicial 169,9 $9 \pm 18,0$, final 142,0 $0 \pm 13,3$ $\mathrm{mmHg}, \mathrm{p}<0,001)$. Lo mismo ocurrió con la PAD tanto en diabéticos (inicial 92,9 $\pm 9,7$, final 78,4 $\pm 8,5 \mathrm{mmHg}, \mathrm{p}<0,001$ ) como en pacientes

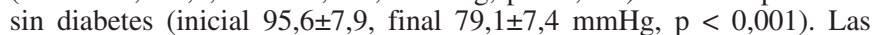
diferencias entre ambos grupos no fueron significativas ni en la visita inicial ni en ninguna de las visitas de revisión. La reducción final obtenida fue -31,7/-14,6 mmHg en diabéticos y -27,6/-16,5 $\mathrm{mmHg}$ en paciente sin diabetes (diferencia sin significación). La reducción final en la presión de pulso no fue diferente entre ambos grupos (diabéticos, 17,8 $\pm 14,5$, frente a pacientes sin diabetes, $11,1 \pm 13,2 \mathrm{mmHg}$ ). Dos pacientes diabéticos necesitaron añadir otro fármaco al tratamiento para conseguir el control de la PA y ninguno en el grupo de pacientes sin diabetes. No se han detectado efectos secundarios.

Conclusiones: El eprosartan demostró ser un fármaco efectivo para reducir ambos componentes de la PA, sin perder eficacia en los pacientes que padecen diabetes mellitus.

PALABRAS CLAVE: Hipertensión arterial. Eprosartan. Diabetes mellitus.
ABSTRACT

Objective: Diabetes mellitus seems to induce an special dificulty to control de la high blood pressure. This effect is more severe on the SBP. Previous reports suggest that a new angiotensin receptor blocker, eprosartan, might have a higher efficacy to reduce SBP. It has been evaluated the BP decrease obtained with eprosartan in a group of diabetics patients compared to non diabetic patients.

Material and methods: 81 patients were recruited of whom 65 have ended follow-up. 34 patients were diabetics (mean age 66.7 \pm 10.7 years, 15 men and 19 women) and 31 were non diabetics control patients (mean age $61.8 \pm 12,8$ years, 13 men and 18 women). All patients were treated with $(600 \mathrm{mg})$ once daily. The dosis was ingested in the morning. They were made three follow up visits (1,3 and 6 mo after the first visit).

Results: SBP was significantly decreased both in diabetics (baseline $170.9 \pm 12.0$, final $139.1 \pm 13.0 \mathrm{mmHg}, p<0.001)$ and in non diabetics group (baseline 169.9 \pm 18.0 , final $142.0 \pm 13.3 \mathrm{mmHg}, p<0.001$ ). DBP was also reduced in both groups (diabetics: baseline 92.9 \pm 9.7 , final $78.4 \pm 8.5 \mathrm{mmHg}, p<0.001$; non diabetics: baseline 95.6 \pm 7.9 , final $79.1 \pm 7.4 \mathrm{mmHg}, p<0.001)$. Differences between the groups were not significant in any visit. Final BP reduction reached was -31.7/-14.6 $\mathrm{mmHg}$ in diabetics vs $-27,6 /-16,5 \mathrm{mmHg}$ in non diabetics patients (difference is not significant) Pulse pressure changes were not different between the twp groups (diabetics, 17.8 \pm 14.5 , vs non diabetics, $11.1 \pm 13.2$ $\mathrm{mmHg}$ ).Two diabetic patients need a second drug to achieve BP goal and no one in non diabetic group. No adverse effects were reported.

Conclusions: Eprosartan seems to be an effective drug to reduce $S B P, D B P$ and pulse pressure with the same effectiveness in diabetics and non diabetic patients.

KEY WORDS: Hypertension. Eprosartan. Diabetes mellitus.

Robles NR, Velasco J, Jimenez Perez, JL, Alonso FJ, Blanco J, Masot J, Matilla C, Nieto J, Romero F, Gamero MJ. Efecto antihipertensivo del eprosartan en pacientes diabéticos. An Med Interna (Madrid) 2004; 21: 223-226.

\section{INTRODUCCIÓN}

Aunque es bien conocida la asociación de la hipertensión con otros factores de riesgo vascular, así como del efecto mul- tiplicativo sobre el riesgo que ocurre cuando los diversos factores están presentes de manera simultánea en el mismo paciente, existe poca información disponible sobre la eficacia relativa del tratamiento antihipertensivo en presencia de otros

Trabajo aceptado: 3 de diciembre de 2003

Correspondencia: Nicolás Roberto Robles Pérez. Sección de Nefrología. Hospital Infanta Cristina. Ctra. de Portugal, s/n. 06080 Badajoz. 
factores de riesgo. Entre estos últimos ocupa un lugar importante la diabetes mellitus, tanto por su prevalencia creciente en la población mundial, como por la importancia del tratamiento hipotensor en la prevención de las complicaciones asociadas al síndrome metadiabético, incluso cuando la presión arterial no está elevada (1-3).

Otro de los problemas cada vez más frecuentes en la práctica clínica es la presencia de hipertensión arterial aislada, cuando la PAD se mantiene normal, o del aumento de la presión de pulso, cuando el aumento de la PAS es superior al de la PAD. Este fenómeno presenta una incidencia cada vez mayor a medida que la población envejece y, lo que es más importante, parece más prevalente en enfermos diabéticos. La experiencia acumulada sugiere que incluso pequeñas elevaciones de la PAS se asocian con un incremento proporcional del riesgo de acontecimientos vasculares del enfermo. La PAS es además mejor predictor de la aparición de estos acontecimientos que la PAD (4-7).

Por otra parte, la respuesta de la PAS a la terapéutica hipotensora es menor que la obtenida sobre el otro componente de la PA, como demuestran numerosos estudios realizados en los últimos años (8). El eprosartan, un antagonista de los receptores de angiotensina de reciente aparición parece ser capaz de obtener una mayor reducción de la PAS que otros fármacos antihipertensivos $(9,10)$. El objetivo del estudio que ahora se describe ha sido valorar la influencia de la presencia de diabetes méllitus sobre el resultado del tratamiento con eprosartan en un grupo de pacientes hipertensos esenciales.

\section{PACIENTES Y MÉTODOS}

Se incluyeron en el estudio los pacientes mayores de 18 años de cualquier sexo que presentaran hipertensión arterial (PAS > $140 \mathrm{mmHg}$ y/o PAD > $90 \mathrm{mmHg}$ confirmada en 3 medidas). Se excluyeron mujeres embarazadas o lactantes o aquellas que no realizaran tratamiento anticonceptivo eficaz. Fueron excluidos los enfermos con PAS $>180 \mathrm{mmHg}$ o PAD $>110 \mathrm{mmHg}$, aquellos que presentaban creatinina plasmática superior a $2 \mathrm{mg} / \mathrm{dl}$, los que tenian antecedentes de alergia o efectos secundarios graves con inhibidores del enzima conversora o antagonistas de los receptores de la angiotensina, asi como los que habian padecido un ACVA, IAM u otra complicación cardiovascular grave en los últimos seis meses.
Se reclutaron para el estudio un total de 81 pacientes de los cuales solamente eran evaluables 65 al final del seguimiento; de ellos, 34 padecían diabetes méllitus y 31 estaban libres de esta enfermedad. No existían diferencias entre ambos grupos en sexo o edad (diabéticos, edad media $66,7 \pm 10,7,15$ hombres y 19 mujeres; no diabéticos edad $(61,8 \pm 12,8,13$ varones y 18 mujeres $)$. Tampoco había diferencias en la PAS, PAD o PP (Tabla I).

En todas las ocasiones la PA fue tomada en dos ocasiones antes de la ingesta de medicación y se halló la media de las dos tomas. La PA y la posible presencia de efectos secundarios fueron valorados a los 30, 90 y 180 días de seguimiento, con una visita opcional a los 60 días si existía mal control de la PA en la primera visita. En el caso de que el paciente estuviera tomando algún fármaco hipotensor previamente, este fue suspendido quince dias antes de iniciar el tratamiento con eprosartan a la dosis de $600 \mathrm{mg}$ en toma única matutina. Los pacientes mal controlados en las visitas de seguimiento podían recibir un segundo fármaco hipotensor, siempre que no fuera un agente que actuase sobre el eje renina-angiotensina.

La comparación de las variables continuas se realizó mediante la prueba de la "t" de Student para datos relacionados. Para las variables de frecuencia se utilizó la prueba de chi cuadrado. Para el estudio estadístico se utilizó el programa informático GST.

\section{RESULTADOS}

La PAS se redujo significativamente tanto en el grupo de diabéticos ( inicial 170,9 $\pm 12,0$, final 139,1 $\pm 13,0 \mathrm{mmHg}, \mathrm{p}<$ 0,001 ) como en el grupo sin diabetes (inicial 169,9 $\pm 18,0$, final 142,0 $\pm 13,3 \mathrm{mmHg}, \mathrm{p}<0,001)$. Lo mismo ocurrió con la PAD

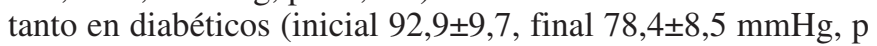
$<0,001$ ) como en pacientes sin diabetes (inicial 95,6 $\pm 7,9$, final 79,1 \pm , $4 \mathrm{mmHg}, \mathrm{p}<0,001)$. Los valores y significaciones correspondientes a las visitas sucesivas han sido recogidas en la tabla I.

Las diferencias entre ambos grupos no fueron significativas ni en la visita inicial ni en ninguna de las visitas de revisión. La reducción final obtenida fue $-31,7 /-14,6 \mathrm{mmHg}$ en diabéticos y $-27,6 /-16,5 \mathrm{mmHg}$ en paciente sin diabetes (la diferencia carece de significación estadística).

TABLA I

EVOLUCIÓN DE LA PA

\begin{tabular}{lcccc}
\hline & Visita 1 & Visita 2 & Visita 3 & Visita 4 \\
\hline DM & $170,9 \pm 12,0$ & $152,0 \pm 12,9$ & $147,5 \pm 15,0$ & $139,1 \pm 13,0$ \\
Sin DM & $169,6 \pm 18,0$ & $151,7 \pm 17,4$ & $148,5 \pm 16,4$ & $142,0 \pm 13,3$ \\
DM & $92,9 \pm 9,7$ & $82,6 \pm 7,7$ & $80,4 \pm 6,8$ & $78,3 \pm 8,5$ \\
Sin DM & $95,6 \pm 7,9$ & $85,8 \pm 9,3$ & $82,9 \pm 8,2$ & $79,1 \pm 7,4$ \\
DM & $77,9 \pm 15,4$ & $69,3 \pm 13,7$ & $67,1 \pm 13,3$ & $60,7 \pm 9,8$ \\
Sin DM & $74,0 \pm 17,9$ & $65,9 \pm 14,6$ & $65,6 \pm 12,5$ & $62,9 \pm 12,0$ \\
\hline
\end{tabular}

Todos los valores expresados como $\mathrm{mmHg}$. Las diferencias entre grupos no son significativas en ningún punto del seguimiento. Los descensos dentro del mismo grupo entre visitas sucesivas son todos significativos $(p<0,01)$. 


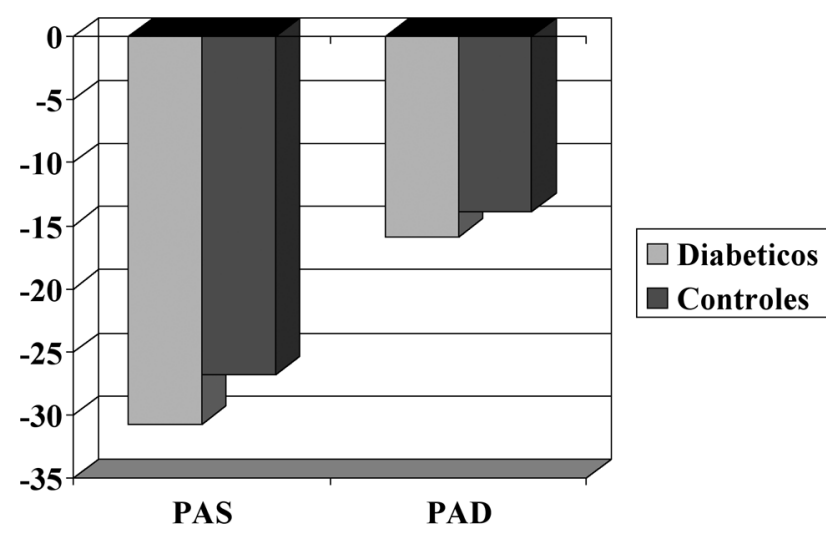

Fig. 1. Descensos finales de la PA. Las diferencias no son significativas.

La presión de pulso se redujo significativamente en ambos grupos desde la primera visita (Fig. 1), sin que tampoco se alcanzaran diferencias entre pacientes diabéticos y pacientes sin diabetes en ninguna de las visitas. La reducción final en la presión de pulso no fue diferente entre ambos grupos (diabéticos, $17,8 \pm 14,5$, frente a pacientes sin diabetes, $11,1 \pm 13,2$ $\mathrm{mmHg}$ ). En ambos grupos se calculó el cociente PP/PAM pero no se detectaron diferencias ni entre diabéticos e hipertensos sin diabetes ni al comienzo del estudio (diabéticos $0,66 \pm 0,14$, no diabéticos $0,61 \pm 0,13$ ) ni al final del estudio

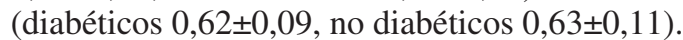

El $94,5 \%$ de los pacientes sin diabetes y el $88,4 \%$ de los diabéticos consiguieron un control óptimo de su PAD $(<90$ $\mathrm{mmHg}$ ) (diferencia sin significación). Solamente el 35,5\% de los no diabéticos y el 41,2\% de los diabéticos alcanzaron una PAS $<140 \mathrm{mmHg}$ al final del tratamiento (no es significativo). Considerando una PAS $<140 \mathrm{mmHg}$, el $61,8 \%$ de los diabéticos alcanzaron buena respuesta frente al 52,6\% de los hipertensos sin diabetes (sin significación estadística).

Dos pacientes diabéticos añadieron otro fármaco al tratamiento para conseguir el control de la PA y ninguno en el grupo de pacientes sin diabetes. No se han detectado efectos secundarios en ninguno de los dos grupos.

\section{DISCUSIÓN}

La hipertensión es un problema más común en los diabéticos que en el resto de la población $(1,2,11)$. Su presencia se asocia además a la aparición de algunas complicaciones del síndrome metadiabético como es la nefropatía diabética (12). Por otra parte, ciertas complicaciones de la hipertensión arterial son también más frecuentes en los diabéticos, como por ejemplo la hipertensión arterial sistólica aislada o el aumento de la presión de pulso $(13,14)$. De esta manera, el tratamiento a relizar en los pacientes que, además, sufren de diabetes mellitus es uno de los temas más importantes de la terapéutica en HTA.

En el momento actual se considera que la medida farmacológica más efectiva en la prevención y el tratamiento de la nefropatía en el diabético es el uso de fármacos que bloqueen el eje renina-angiotensina. En los diabéticos tipo I se recomienda que este bloqueo se realice con inhibidores del enzima conversora de la angiotensina, mientras que la experiencia acumulada con antagonistas de los receptores de la angiotensina aconsejan que sean estos últimos los usados en enfermos diabéticos tipo II, este sería el caso del eprosartan, el fármaco utilizado en este estudio $(12,15)$.

Diversos estudios sugieren que la presencia de diabetes confiere una especial dificultad para alcanzar los objetivos terapéuticos deseados, especialmente cuando presentan aumento de la presión de pulso al comienzo del tratamiento $(1,16)$. En nuestra experiencia, la presencia de diabetes mellitus no modificó el grado de respuesta hipotensora al tratamiento con eprosartan, tanto si nos referimos a los componentes clásicos de la PA, sistólica y diastólica, como en lo que respecta a la presión de pulso. Tampoco hubo diferencia en el número de pacientes respondedores a este respecto. Puesto que los niveles de PA eran similares en ambos grupos al comienzo del estudio, la homogenización de la PP utilizando su cociente con la PAM como se ha realizado en otras ocasiones (10) no ha originado ningún cambio en los resultados obtenidos.

Mientras que la PAD disminuye a partir de los 55 años y pierde gran parte de su capacidad predictiva del riesgo cardiovascular del paciente, la PAS y el PP continúan creciendo, de tal manera que en el paciente anciano (> 65 años) la forma más común de HTA es la hipertensión arterial sistólica aislada. Además, tanto la PAS como la PP son más eficaces para predecir el riesgo de aparición de acontecimientos vasculares en los pacientes de esta edad (4-7). Por todo ello, la evaluación del efecto de los fármacos sobre la presión de pulso es también un tema de candente interés en este momento.

El tratamiento del aumento de la PAS es un problema actualmente desde el punto de vista terapéutico (17). Buena parte de los estudios de intervención publicados hasta ahora no fueron capaces de obtener el objetivo deseado de presión arterial sistólica a pesar del esfuerzo terapéutico realizado (8). Este ha sido también el caso en nuestros resultados, donde el número de pacientes que lograron alcanzar el objetivo de PAS $(<140 \mathrm{mmHg}$ ) fue muy inferior al de aquellos que consiguieron normalizar su PAD. Aunque un número importante de pacientes deberían haber recibido más de un fármaco según el protocolo este no se añadió en la mayoría de los casos traduciendo una vez más el modo de tratamiento usual en la práctica clínica habitual. La necesidad de concienciar a los médicos, y particularmente a aquellos del nivel de atención primaria, de la obligación de aumentar el tratamiento cuando existe un control subóptimo de la PA se muestra una vez más importante.

Entre los factores de riesgo que se asocian al aumento de la PAS y la PP la diabetes mellitus ocupa un lugar destacado por encima del tabaquismo y la dislipemia $(4,13,14)$. Existe evidencia científica que sugiere que el tratamiento del aumento de la presión de pulso puede ser más difícil en diabéticos que en el resto de la población $(18,19)$. Este es el caso del estudio INSIGHT, en el cual el subgrupo de diabéticos, aproximadamente una sexta parte de la muestra, necesito mayor número de fármacos para alcanzar la reducción deseada de la PAS a pesar de partir de niveles de PAS similares (20). No ha sido este el caso. Nuestros resultados sugieren una eficacia similar en la reducción de la PAS y la $\mathrm{PP}$ en ambos grupos, diabéticos y no diabéticos. En este 
sentido, la ausencia de efecto de la diabetes mellitus sobre la respuesta terapéutica al eprosartan ha sido demostrada en otros estudios.

El tipo de fármaco utilizado en el tratamiento puede ser importante. Se conocen buenos resultados en el tratamiento de la HTA sistólica con diuréticos y con calcioantagonistas $(21,22)$. La evidencia actual con antagonistas de receptores de la angiotensina es relativamente escasa, pero el eprosartan ha demostrado ser superior al enalapril en la reducción de la PAS (9). En este estudio no se ha realizado ninguna comparación con otro fármaco pero la respuesta obtenida puede ser considerada como muy satisfactoria en comparación con otros estudios previamente publicados.

Aunque la mayor parte de las guías clínicas consideran ya un valor objetivo de PA en diabéticos inferior al aconsejado para la población que no padece diabetes, en el diseño de los objetivos se ha conservado el mismo valor para ambos grupos a fin de poder comparar la tasa de respondedores $(15,23)$. No obstante, la reducción adecuada de las cifras tensionales de los diabéticos de acuerdo a los objetivos indicados por los consensos internacionales debe ser un objetivo irrenunciable de la práctica clínica.

El eprosartan ha demostrado ser un fármaco eficaz para la reducción de la presión arterial sistólica, diastólica y de la presión de pulso. La presencia de diabetes no modificó la respuesta obtenida sobre las cifras de presión arterial. El fármaco presenta un buen perfil de seguridad y escaso número de efectos secundarios.

\section{Bibliografía}

1. Divison Garrote JA, Artigas Rodenas LM. El control de la presión arterial del paciente diabético hipertenso. Hipertensión 2002; 19: 335-337.

2. Sowers JR, Epstein M. Diabetes mellitus and associated hypertension, vascular disease and nephropathy: An update. Hypertension 1995; 26: 869-879.

3. Padwal R, Strauss SE, McAlister FA. Cardiovascular risk factors and their effects on the decision to treat hipertensión: Evidence based review. Br Med J 2001; 977-980.

4. Van Bortel LMAB, Struijker-Boudier HAJ., Safar ME. Pulse Pressure, Arterial Stiffness, and Drug Treatment of Hypertension. Hypertension 2001; 38: 914-921.

5. Sagie A, Larson MG, Levy D. The natural history of borderline isolated systolic hypertension. N Engl J Med 1993; 329: 1912-1917.

6. Franklin SS, Khan SA, Wong ND, Larson MG, Levy D. Is pulse pressure useful in predicting risk for coronary heart disease: The Framingham Heart Study. Circulation 1999; 100: 354-360.

7. Benetos A, Zureik M, Morcet J, Thomas F, Bean K, Safar M, Ducimetiere P, Guize L. A decrease in diastolic blood pressure combined with an increase in systolic blood pressure is associated with high cardiovascular mortality. J Am Coll Cardiol 2000; 35: 673-680.

8. Mancia G. J. Hypertens 2002; 20: 1461-1464.

9. Sega R. Efficacy and safety of eprosartan in severe hypertension. Eprosartan Multinational Study Group. Blood Pressure 1999; 8: 114-121.

10. De la Sierra A, Muñoz A, Arcos E, Lopez JS, Relats J en representación de los investigadores del estudio ETAPA-2. Efecto del tratamiento con eprosartan sobre la presión de pulso. Factores predictores de la respuesta. Hipertensión 2003; 20: 56-62.

11. The National High Blood Pressure Education Program Working Group. National High Blood Pressure Education Program Working Group report on hypertension in diabetes. Hypertension. 1994; 23: 145-158.

12. Robles NR. Nuevas evidencias en nefropatia diabética. Rev Clin Esp 2002; 202: 599-601.

13. Corman B, Duriez M, Poitevin P, Heudes D, Bruneval P, Tedgui A, Levy BI. Aminoguanidine prevents age-related arterial stiffening and cardiac hypertrophy. Proc Natl Acad Sci USA 1998; 95: 1301-1306.
14. Wolffenbuttel BH, Boulanger CM, Crijns FR, Huijberts MS, Poitevin P, Swennen GN, Vasan S, Egan JJ, Ulrich P, Cerami A, Levy BI. Breakers of advanced glycation end-products restore large artery properties in experimental diabetes. Proc Natl Acad Sci USA 1998; 95: 4630-4634.

15. American Diabetes Association (ADA). Standard of medical care for patients with diabetes mellitus. Diabetes Care 2002; 25 (Supl. 1): S33S49.

16. Sowers J, Reed J. Clinical advisory treatment of hypertension in diabetes. J Clin Hypertens 2000; 2: 132-133.

17. De la Sierra A, Artes M, Lopez JS, Arcos E, Muñoz A en representación de los investigadores del estudio ETAPA-2. Efecto del tratamiento con antihipertensivo sobre la presión de pulso. Med Clin (Barc) 2002; 119: 41-45.

18. UK Prospective Diabetes Study Group. Tight blood pressure control and risk of macrovascular and micovascular complications in type 2 diabetes. BMJ 1998; 317: 703-713.

19. Hansson L, Zanchetti A, Carruthers SG, Dahlof B, Elmfeldt D, Julius S, et al for the HOT Study Group. Effect on intensive blood pressure lowering and low dose aspirin in patients with hypertension: Principal results of the Hypertension Optimal Treatment (HOT) randomized trial. Lancet 1998; 351: 1755-1762.

20. Brown MJ, Castaigne A, de Leeuw P, Mancia G, Palmer CR, Rosenthal T, Ruilope LM. Influencia de la diabetes y el tipo de hipertensión arterial en la respuesta al tratamiento antihipertensivo. Hypertension 2000; 35: 1038-1042.

21. Tuomilehto J, Rasteyne D, Birkenhager WH, Thijs L, Antikainen R, Bulpitt CJ, et al. Effects of calcium-channel blockade in older patients with diabetes and systolic hypertension. N Engl J Med 1999; 340: 677-684.

22. Cushman WC, Masterson BJ, Williams DV, Reda DJ, for the Veterans Affair Cooperative Study Group on Antihypertensive Agents. Pulse pressure changes with six classes of antihypertensive agents in a randomized, controlled trial. Hypertension 2001; 38: 953-957.

23. Guidelines Committee. 2003 European Society of Hypertension-European Society of Cardiology guidelines for the management of arterial hypertension. J Hypertension 2003; 21: 1011-1053. 